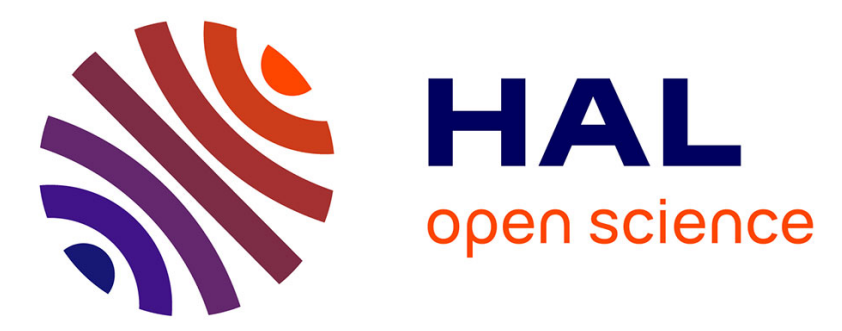

\title{
User Influence on Two Complementary Residual Stress Determination Methods: Contour Method and Incremental X-Ray Diffraction
}

Bruno Levieil, F. Bridier, Cédric Doudard, David Thevenet, Sylvain Calloch

\section{- To cite this version:}

Bruno Levieil, F. Bridier, Cédric Doudard, David Thevenet, Sylvain Calloch. User Influence on Two Complementary Residual Stress Determination Methods: Contour Method and Incremental X-Ray Diffraction. Experimental Mechanics, 2016, 56 (9), pp.1641-1652. 10.1007/s11340-016-0189-3 . hal01701211

HAL Id: hal-01701211

https://hal-ensta-bretagne.archives-ouvertes.fr/hal-01701211

Submitted on 24 Sep 2021

HAL is a multi-disciplinary open access archive for the deposit and dissemination of scientific research documents, whether they are published or not. The documents may come from teaching and research institutions in France or abroad, or from public or private research centers.
L'archive ouverte pluridisciplinaire HAL, est destinée au dépôt et à la diffusion de documents scientifiques de niveau recherche, publiés ou non, émanant des établissements d'enseignement et de recherche français ou étrangers, des laboratoires publics ou privés.

\section{(c)(1)}

Distributed under a Creative Commons Attribution| 4.0 International License 


\title{
User Influence on Two Complementary Residual Stress Determination Methods: Contour Method and Incremental X-Ray Diffraction
}

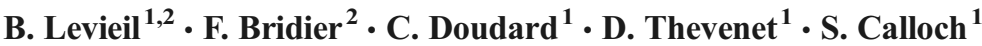

\begin{abstract}
The combination of various residual stress measurement methods is a common practice to complete knowledge that a single measurement method cannot provide. In this study, incremental X-Ray diffraction is combined with the contour method to measure a bent notched specimen to study the methods robustness. A finite element analysis model is built and validated with strain measurement of the bending process thus providing prior knowledge of the residual stress field. Three-dimensional neutron diffraction residual stress measurements are also performed to obtain a reference measurement with a non-destructive method and to validate the simulated stress field. In-depth stress gradient measured by Xray diffraction is corrected with four different methods that all show good correlation with neutron diffraction measurements. Correction methods, assumptions and uncertainties are discussed and differences are observed on the robustness of the methods. Contour method measurements are performed and results are also in agreement with neutron measurements. The results provided by the contour method are complementary to those of the X-Ray diffraction since, despite a lower accuracy on the edges where X-ray diffraction is performed, the contour method offers the complete cartography of longitudinal stress in a symmetry plane of the bent specimen. Uncertainty of the contour method due to the postprocessing procedure is discussed.
\end{abstract}

B. Levieil

Bruno.levieil@ensta-bretagne.org

1 Institut de Recherche Dupuy de Lôme (FRE 3744), UBS/UBO/ ENSTA-Bretagne/ENIB, 2 rue François Verny, 29806 Brest, Cedex 9, France

2 DCNS Research, - TCO - 5, rue de l'Halbrane, 44340 Bougenais, France
Keywords Residual stresses $\cdot$ Contour method $\cdot$ X-Ray diffraction $\cdot$ Neutron diffraction $\cdot$ Stress relaxation correction method

\section{Introduction}

Residual stresses (RS) may have a strong influence on the mechanical resistance of parts $[1,2]$ and thus need to be characterized. However, the measurement of residual stresses remains a challenging problem as no unique method exists. Each method has its own advantages and limitations in terms of gauge volume, accuracy, depth penetration, and material microstructure [3]. Combining methods is a solution either to obtain different information, or to validate the assumptions and approximations of each measurement technique. Combining a diffraction method and a relaxation based method is also interesting as their fundamental measurement principle is not the same. If all methods aim at measuring local stresses through local strains, X-ray Diffraction (XRD) uses lattice spacing by measuring diffracting peak shift whereas relaxation methods such as the contour method measure part deformation at the meso-scale (i.e., scale of the specimen) disregarding thereby variation at the micro-scale.

This study aims at comparing two complementary measurement methods' robustness: Incremental X-Ray Diffraction (XRD) and Contour Method (CM) and especially focuses on the robustness of these methods regarding both hypothesis and post-processing procedures.

Incremental XRD involves material removal and thus RS relaxation $[4,5]$. Different methods exist to correct that relaxation but they all require some hypotheses. The first hypothesis that all methods use is that the stress relaxation is elastic. The Moore and Evans analytical method also supposes simple geometrical cases that can be a strong assumption. Numerical 
methods exist for complex geometries but require the prior estimation of the initial RS field. The robustness of the methods regarding the supposed initial stress field is also studied in this work to quantify the robustness of the different stress relaxation correction methods.

The second RS measurement method that is studied here is the contour method which is often used as a complementary method to XRD to complete RS knowledge in other directions $[6,7]$ or to confirm measurements $[8,9]$ combining a diffraction and a relaxation method to measure the whole stress gradient in a symmetry plane of the part. CM is based on the measurement of the displacement due to stress relaxation on a cut face. Displacement measurements are then smoothed and interpolated on a finite element model of the part. RS results are thus subjected to data processing. Evaluation of the robustness of the postprocessing process is carried out in this work.

In this application, these two methods are applied on a notched specimen that has been bent and relaxed to introduce RS. The first step of this work is to obtain the best knowledge of the RS stress field. A finite element analysis of the bending process is carried out and contact between the rollers and the specimen is calibrated through experimental strain measurements. To validate the predicted stress field, Neutron Diffraction (ND) stress measurements are then performed as this technique has the capability to measure the full stress tensor below the surface without any material removal.

Then, incremental XRD is performed and the four correction methods are used, analyzed and method assumptions are tested.

Finally, CM is applied, the experimental procedure is described and a post-processing procedure is proposed to evaluate post-processing uncertainty.

\section{Case Study}

\section{Geometry}

Specimen material is machined from a high-strength ferritic steel isotropic sheet. The material does not exhibit any evidence of texture. In order to introduce residual stresses (RS) around the notches, a bending load is applied as shown in Fig. 1 After unloading the specimen, tensile RS are expected to appear around one notch which will be referred to as the "tensile" notch and compressive $\mathrm{RS}$ on the other notch which will be referred to as the "compressive" notch. Two specimens were necessary for this study as destructive measurements techniques are employed. They were machined in the same sheet and bent in the same conditions to minimize experimental variations.

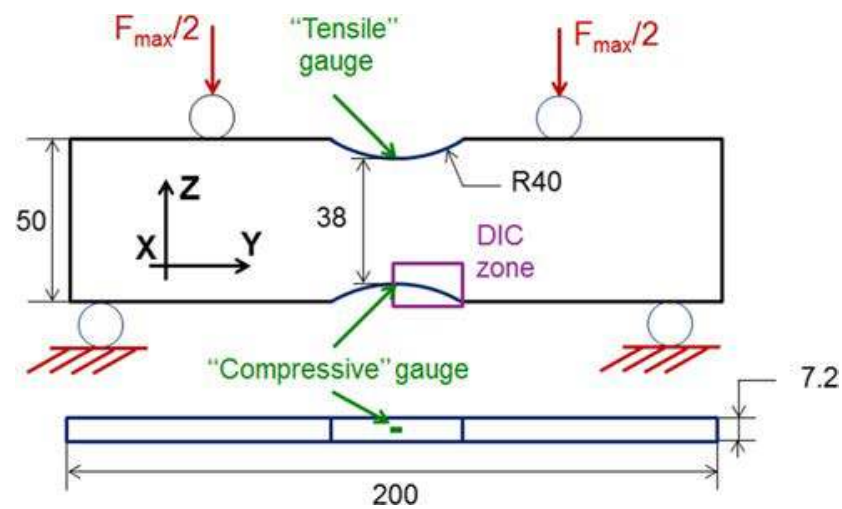

Fig. 1 Specimen geometry and bending set-up

\section{Finite Element Prediction of Residual Stresses}

A load-unload test is used for the material characterization. Such tests are useful for the prediction of residual stress state since they particularly inform on the evolution of the elastic domain during unloading [10]. The design of the sample used for the load-unload test is shown in Fig. 2.

The load-unload test consists in a tensile loading test with several reverse compression loadings at increasing plastic strain levels as illustrated in Fig. 3. Stresses are normalized to the initial tensile elastic limit $\sigma_{\mathrm{y}}$ and strains to the initial elastic deformation limit $\varepsilon_{\mathrm{y}}=\mathrm{E} / \sigma_{\mathrm{y}}$ where $\mathrm{E}$ is the Young's modulus of the material. At each unload, the specimen load

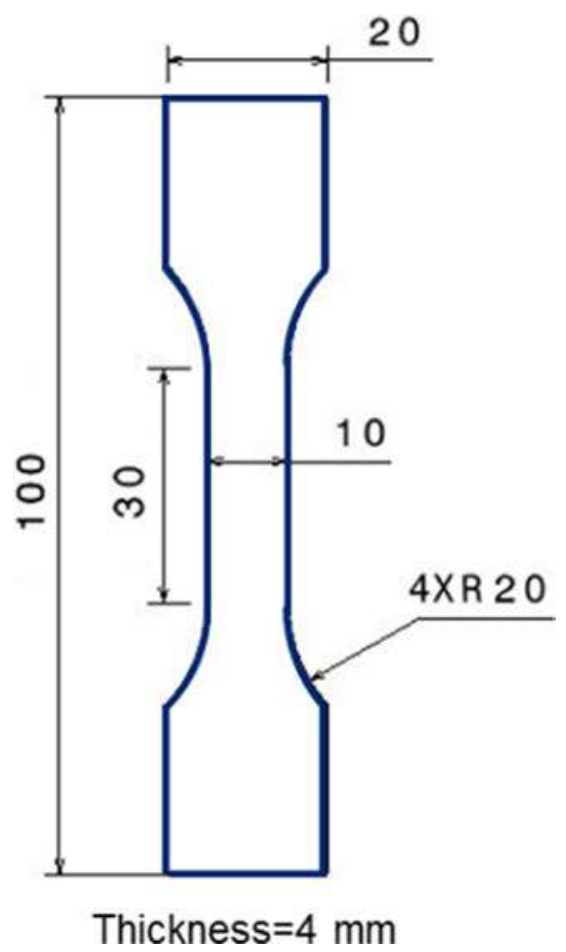

Fig. 2 Tensile specimen 
Fig. 3 Load-unload test used for material characterization

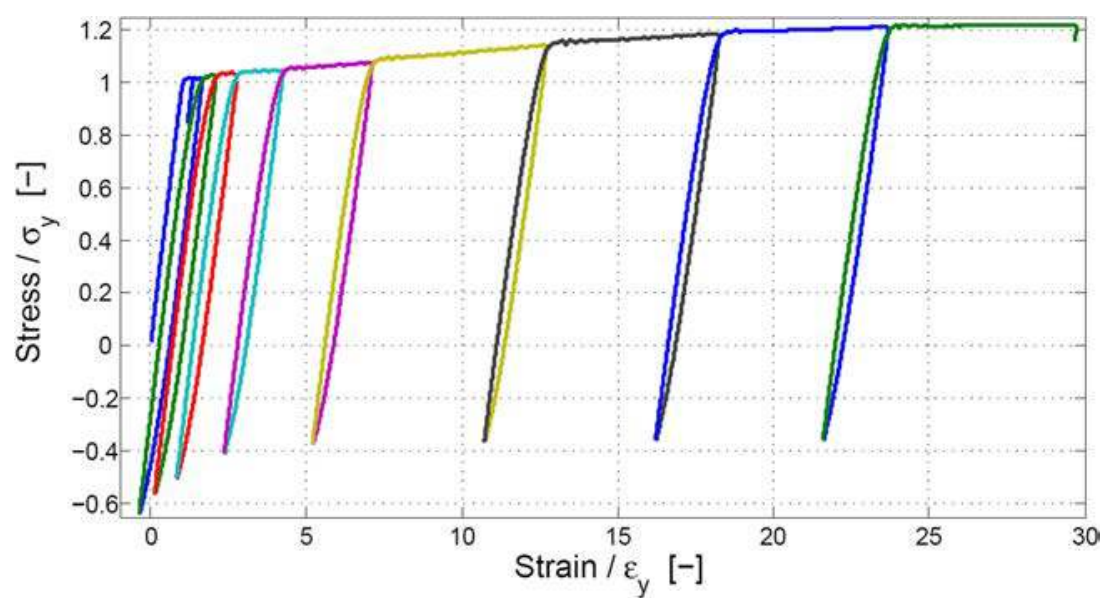

varies from the tensile elastic limit to the compressive elastic limit of the material giving thus the elastic domain evolution. The knowledge of the center respectively the size, of the elastic domain permits the identification of the kinematic, respectively the isotropic, hardening evolution with plastic strain. Isotropic hardening is modeled by the Voce model [11] and the kinematic hardening by the Armstrong-Frederick model [12].

Part geometry is modeled using two symmetries with normal directions X and Y. The mesh consists of 200,000 linear hexahedral elements of type C3D8R (Abaqus ${ }^{\circledR} / \mathrm{CAE}$ ).

Interaction between rollers and specimens has to be taken into account in the FEA as the bending load is important (it leads to matting of the specimen behind the rollers). This deformation constrains the specimen and adds a tensile load to the bending moment that has to be considered. Rollers are considered as a rigid body and friction is modeled by a linear model, the coefficient of which is calibrated with the strain information previously obtained.

Figure 4 shows the stress solutions that are obtained for five different friction coefficients from 0 (no friction) to 1: longitudinal translation (along $\mathrm{Y}$ direction) of elements in contact with the roller is impossible.
Calibration of the friction coefficient is necessary as various values give different stress fields. For that purpose, strains are measured during the experimental bending process.

\section{Displacement Measurements}

KYOWA® KFG-02-120-C1-11 strain gauges are used with a measurement surface of $0.2 \times 1.3 \mathrm{~mm}^{2}$ at each notch as shown in Fig. 1 to access the local maximum strain value during loading. Digital image correlation (DIC) is accomplished using a GOM $5 \mathrm{M}{ }^{\circledR}$ device to measure the strain field near the "tensile" notch. The measurement area is a $15 \times 13 \mathrm{~mm}^{2}$ zone shown in Fig. 1. The interest in combining the two techniques is to have more precise yet local information with the strain gauge whereas DIC offers the strain field distribution on a larger zone. The measurements show good agreement and the strain measured at the "tensile" notch is $0.8 \%$ at the maximum bending load Fmax and a residual strain of $0.26 \%$ after unloading the specimen as shown in Fig. 5 .

Using the strain information measured allows the discrimination of the FEA friction coefficient $f$.
Fig. 4 Residual stress gradient after unloading predicted by FEA for the different stress coefficients

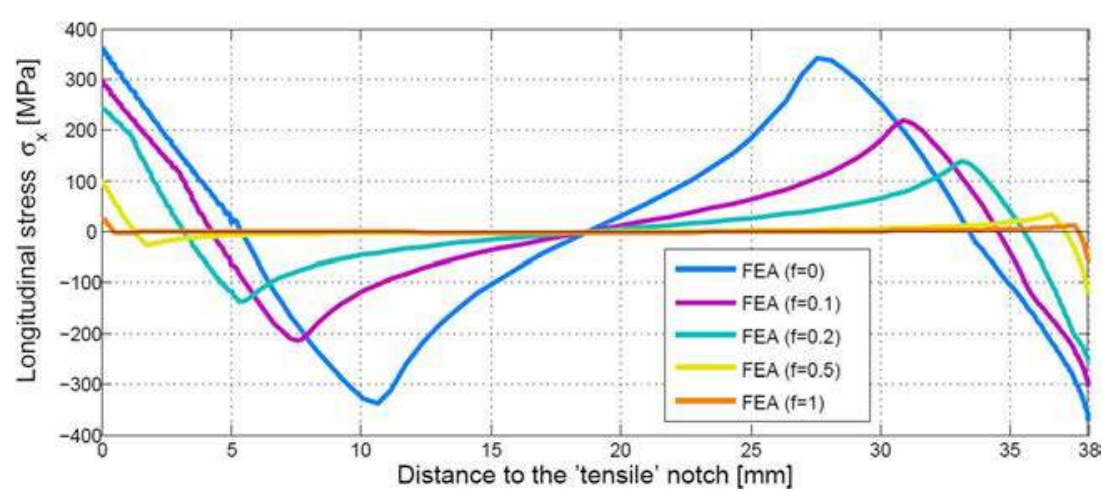


Fig. 5 Dimensionless bending force F/Fmax vs. strain curves comparison between strain gauges, DIC \& FEA

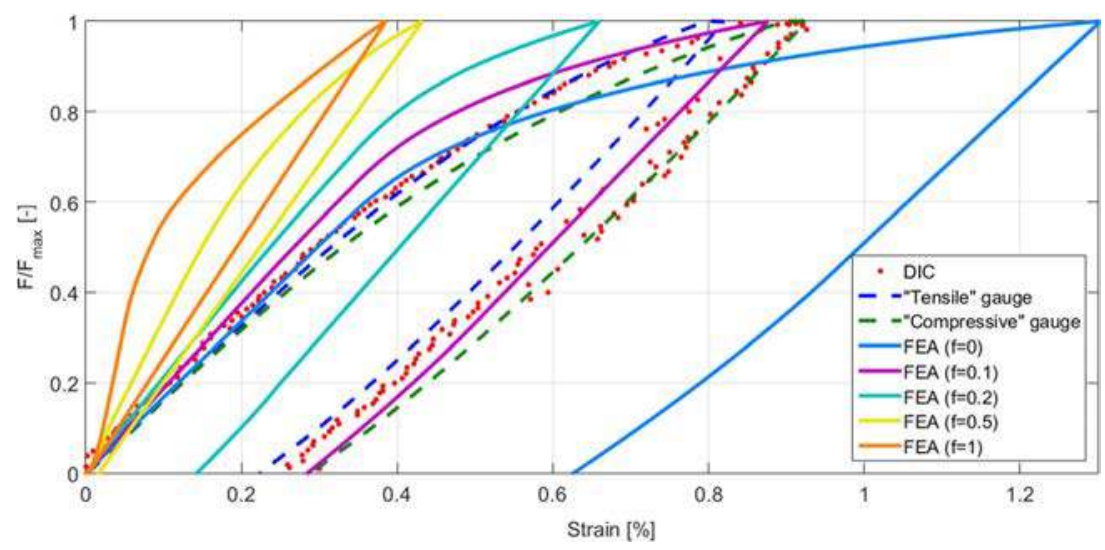

Figure 5 shows the evolution of the local strain on the notch during bending and unloading. It indicates that $f$ should be taken slightly lower than 0.1 .

Figure 6 shows the strain gradient away from the notch and confirms the previous result.

A value of $f=0.1$ is chosen for the FEA. Validation of the global strain field is shown in Fig. 7 showing good agreement both at maximum bending load (Fig. 7a) and after unloading (Fig. 7b) between the global experimental strain field on the left and the predicted strain field on the right.

Stress gradient evolution for $f=0.1$ can now be observed in Fig. 4. It shows that the longitudinal stress at the notches should be $\pm 300 \mathrm{MPa}$ after unloading. Stress sign reverses after $4 \mathrm{~mm}$ below the surface of the notches and maximum subsurface stress is $\pm 215 \mathrm{MPa}$ located $7.5 \mathrm{~mm}$ away from the notches. According to FEA, the stress profile has a perfect central symmetry. Stress prediction validation is now performed using Neutron Diffraction (ND)

\section{Neutron Diffraction Measurements}

ND measurements were performed at the DIANE (G52) line of the Laboratoire Léon Brillouin of the CEA Saclay. Seven measurement points in the three main directions were established with a gauge volume of $2 \mathrm{~mm}$ in $\mathrm{X}$ and $\mathrm{Y}$ directions where stresses are almost constant and reduced to $1 \mathrm{~mm}$ in the $\mathrm{Z}$ direction where the stress gradient is steep. The 21 peak shifts were obtained with the fitting of the intensity signals of the $\{211\}$ crystalline planes corresponding to those of the ferrite. In all this study, the strain values are converted into stresses using Hooke's law for isotropic materials. Elastic parameters were taken from the literature [13] for a $\mathrm{Cr}-\mathrm{K} \alpha$ source and the $\{211\}$ crystallographic plane of ferritic steels $\left(\mathrm{S}_{1}=-1.2510^{-6} \mathrm{MPa}^{-1}\right.$ and $\left.1 / 2 \mathrm{~S}_{2}=5.7610^{-6} \mathrm{MPa}^{-1}\right)$. A reference value was adjusted so as to respect the autoequilibrium of the stress profile. Results are illustrated in Fig. 8 and compared to FEA prediction. Horizontal error bars on the neutron measurements correspond to the $1 \mathrm{~mm}$ gauge volume in the $\mathrm{Z}$ direction whereas vertical error bars correspond to the $\pm 2 \sigma$ error bars on the peak fitting, where $\sigma$ is the standard deviation.

Neutron measurements which will be considered as the experimental reference in this study showed a good correlation with FEA, especially in the Y direction which is the principal stress direction. For the rest of this study, only stresses in $\mathrm{Y}$ direction will be considered significant. An edge effect is observed at the first measurement point which is probably due to local machining residual stresses introduced before
Fig. 6 Strain gradient comparison between DIC \& FEA with different $f$ values a) At maximum bending load

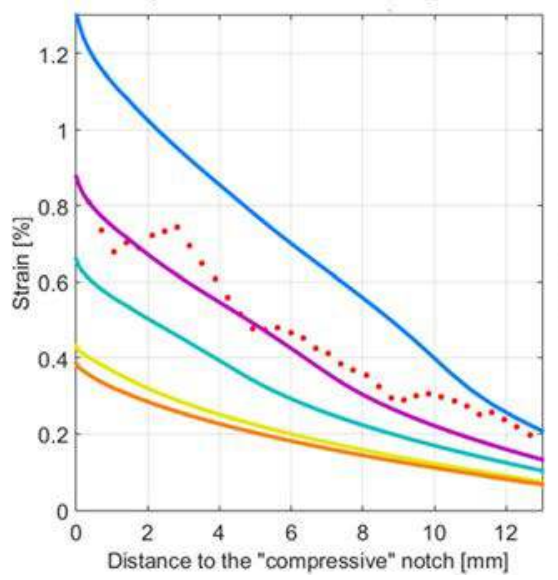

b) After unloading

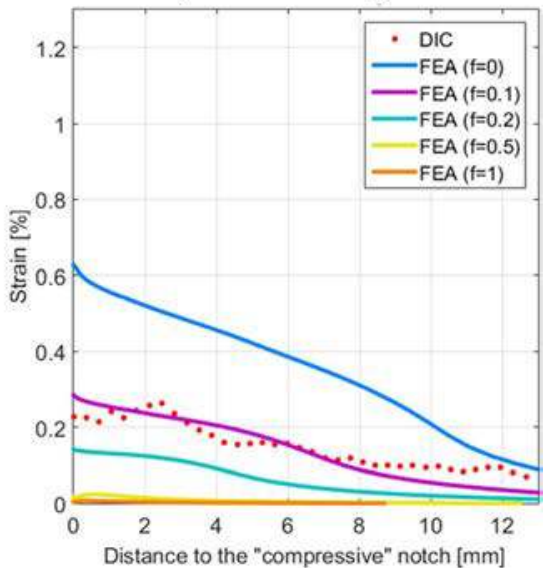


a) At maximum bending load

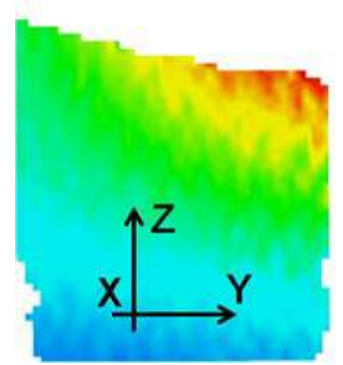

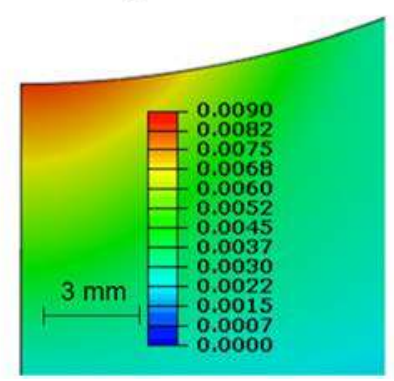

b) After unloading
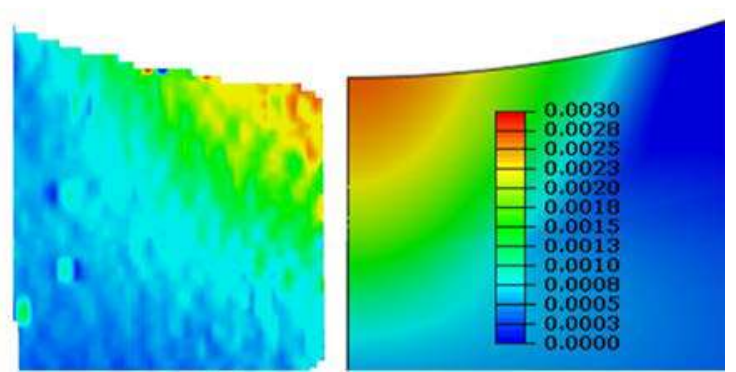

Fig. 7 Strain maps comparison between DIC (left) \& FEA (right) at maximal bending force (a) and after unloading (b)

bending as shown by XRD measurements in the following section. The inversion point (where stresses become compressive) seems to be just before $4 \mathrm{~mm}$ which agrees with FEA. The maximum compressive stress is $-180 \mathrm{MPa}$ at $7.5 \mathrm{~mm}$ whereas FEA predicts a maximum compressive stress of $215 \mathrm{MPa}$ at $7.5 \mathrm{~mm}$.

\section{XRD Measurements \& Correction Methods}

\section{Measurement Conditions}

X-Ray diffraction (XRD) measurements are performed with a Set-X ELPHYSE device (54 kVA) using the $\sin ^{2} \psi$ method [2] and 19 tilt angles. The elastic constants taken for the stress calculation are the same as the ones taken for neutron diffraction. XRD gauge volume was approximately a spot of $1 \mathrm{~mm}$ diameter on the surface and the penetration is estimated to be $10 \mu \mathrm{m}$ in the $\mathrm{Z}$ direction. Gauge volume is thus 100 times smaller in the stress gradient direction, providing better resolution than ND. However, the X-ray measurement can only be practiced on the surface. In order to measure in-depth stresses, 23 polishing steps were practiced up to $5.25 \mathrm{~mm}$ below the surface. After each layer removal step, RS measurement is performed. Electropolishing was employed as this method does not introduce residual stress nor induces preferential grain boundary etching [14].

After each polishing step, RS are redistributed in the specimen to respect the auto-equilibrium of the stress profile.
Therefore, measured RS differ from the original RS. Four existing correction methods using different hypotheses or principles are now compared.

\section{Moore-Evans Analytical Correction Method}

The first method proposed is the Moore \& Evans (ME) stress relaxation method [15] that consists in a set of analytical formulas calculated for three simple geometrical cases: cylinder, tube and plate. It has two main assumptions: stress redistribution is purely elastic and geometries are perfect. Resolving the equilibrium and compatibility equations, one can calculate the corrected stress profile that is supposed to be the original profile.

In comparison to the bent test specimen, the closest geometrical situation described by ME is considered to be the flat plate case. Using the flat plate equation, each term of the corrected in-plane stress vector $\underline{\sigma^{c}}$ can thus be written as

$\sigma^{c}\left(Z_{1}\right)=\sigma^{m}\left(Z_{1}\right)+2 \int_{z_{1}}^{H} \frac{\sigma^{m}(z) d z}{z}-6 Z_{1} \int_{z_{1}}^{H} \frac{\sigma^{m}(z) d z}{z^{2}}$

where $Z_{1}$ is the remaining thickness of the plate in the polishing direction, $\sigma^{m}$ is the measured in-plane stress vector and $\mathrm{H}$ is the total specimen thickness as illustrated in Fig. 9 that also shows the necessary geometry simplification for the application of the ME method. The :, respectively, notations are used for vectors, respectively for matrixes.
Fig. 8 3D neutron diffraction RS measurements compared to FEA prediction

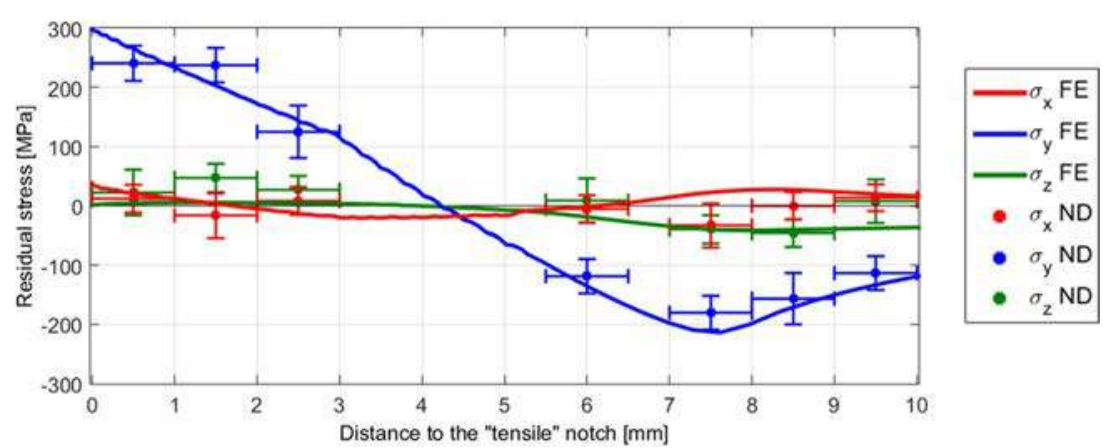




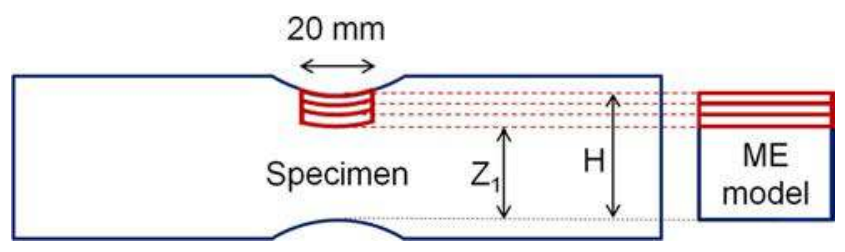

Fig. 9 Geometry assumption for the application of the Moore \& Evans method

\section{Finite Elements Analysis Correction Methods}

Finite element analysis (FEA) stress relaxation methods are based on the fact that the relaxation of a fictive stress profile is the same as the experimental real profile as long as a few hypotheses are assumed. Thus, by numerically simulating the stress relaxation on a fictive profile when removing polished elements from the simulation, a lower triangular relaxation matrix $\underline{\underline{\sigma^{f}}}$ is obtained where each term $\sigma_{d s}^{f}$ is the fictive stress at the top of layer $d$ after the polishing step $s$. This matrix can also be considered as the concatenation of the remaining stress profile column vectors after each polishing step. The same matrix can be built with the experimental profile although only diagonal terms are known (in the dashed ellipse) as they can be experimentally measured by XRD. The objective of all FEA methods is to reconstruct the original experimental profile which is the first column of the experimental relaxation matrix $\underline{\underline{\sigma^{m}}}$ that is supposed to be the real profile, if the XRD measurement error is ignored. The two matrixes are illustrated in Fig. 10, where $D$ is the maximum depth polished.

The correction proposed by Pedersen \& Hansson (PH) (1989) [16] is a direct proportional correction where each corrected stress at depth $d$ can be expressed as

$\sigma_{d}^{c}=\sigma_{d}^{m} \cdot \sigma_{d}^{p}$,

where $\sigma_{d}^{p}=\frac{\sigma_{d d}^{f}}{\sigma_{d 1}^{f}}$ is the proportion of released fictive stress between the original fictive stress $\sigma_{d 1}^{f}$ at depth $d$ and the released stress when all elements above depth $d$ have been deactivated from the model $\sigma_{d d}^{f}$.

The Lambdatech method (1996) [17] is also a numerical method but instead of applying a direct correction, measurements are corrected taking into account the redistribution step after step

$\underline{\sigma}^{c}=[\underline{K}+\underline{I}] \cdot \underline{\sigma^{m}}$

where $\underline{\underline{I}}$ is the identity matrix and $\underline{\underline{K}}$ is a lower triangular matrix in which each coefficient can be calculated as

$K_{d s}=\frac{\sigma_{d s-1}^{f}-\sigma_{d s}^{f}}{\sigma_{d d}^{f}}$

The Savaria-Bridier-Bocher (SBB) method [18] is an improvement of the Lambdatech method that instead of considering the stress on the top of the removed layer as the stress in the whole layer, averages the stress on the top of the removed layer with the stress at the top of the next layer i.e. the stress at the bottom of the removed layer. The previous formula becomes

$\underline{\sigma^{c}}=\left[\underline{K^{\prime}}+\underline{I}\right] \cdot \underline{\sigma_{a v g^{\prime}}^{m}}$

where each term $K_{d s}^{\prime}$ is expressed as

$K_{d s}^{\prime}=2 \cdot \frac{\sigma_{d s-1}^{f}-\sigma_{d s}^{f}}{\sigma_{d d}^{f}+\sigma_{d+1 s+1}^{f}}$

and

$\sigma_{a v g d}^{m}=\frac{\sigma_{d d}^{m}+\sigma_{d+1 d+1}^{m}}{2}$

\section{Results}

XRD raw measurements compared with the four corrected data are presented in Fig. 11. Raw results (red line) bring to light the machining edge effects with a surface stress of $65 \mathrm{MPa}$ that evolve after $70 \mu \mathrm{m}$ into a $250 \mathrm{MPa}$ stress which is very close to the ND value at $0.5 \mathrm{~mm}$. After $2 \mathrm{~mm}$, raw XRD results diverge from ND measurements and after $5.25 \mathrm{~mm}$ raw XRD results are still positive whereas ND results indicate a change of longitudinal stress sign at $4 \mathrm{~mm}$ away from the notch.
Fig. 10 Difference between the fictive stress relaxation matrix and real matrix

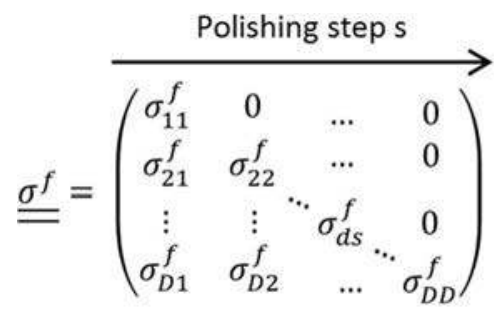

1) Fictive stress relaxation matrix

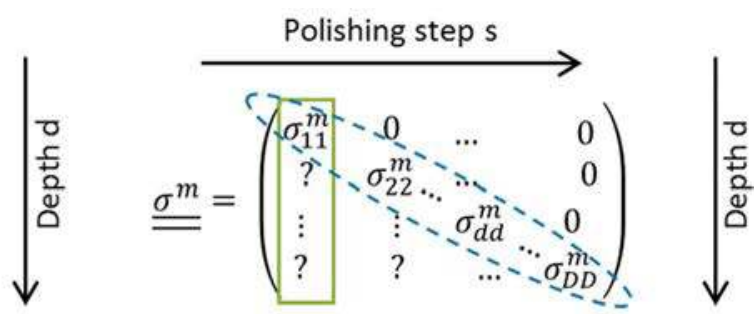

2) Real/experimental stress relaxation matrix 
Fig. 11 Raw and corrected XRD results compared to ND measurements and FEA

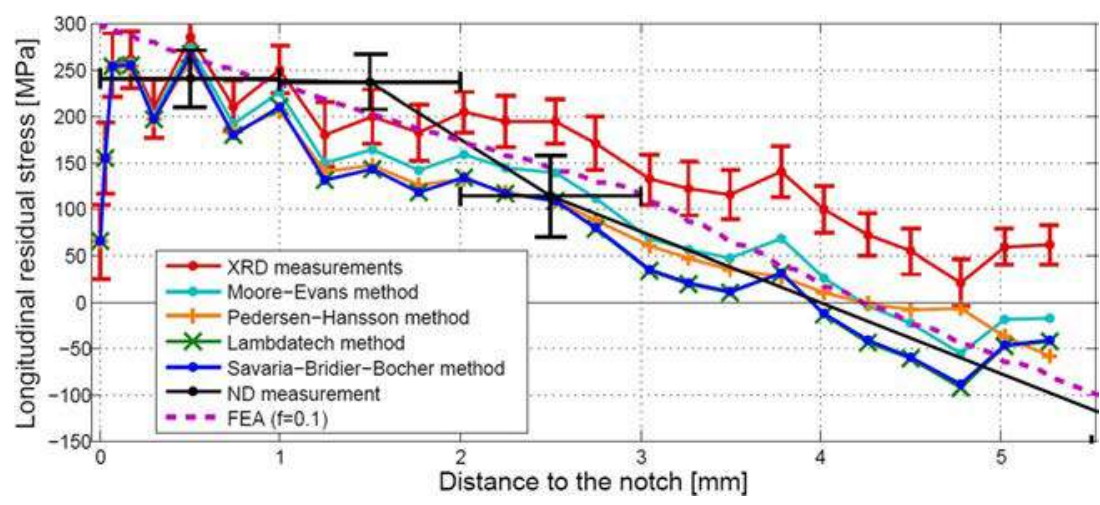

The FEA correction methods have been applied using the bending simulation FEA results as the fictive stress field. The four correction methods improve XRD results as they are closer to the ND measurement than uncorrected results. Longitudinal stress signs change between 3.9 and $4.5 \mathrm{~mm}$ according to the correction method. The correction effect becomes non negligible when compared to measurement scattering after $1 \mathrm{~mm}$ where correction is more than $10 \%$ of the measured XRD value. This value strongly depends on stress gradient and remaining material volume $[4,14,15,19]$. Stress correction rises to $80 \mathrm{MPa}$ for the Moore and Evans method and up to $120 \mathrm{MPa}$ for FEA methods.

Lambdatech and Savaria-Bridier-Bocher methods give very similar results with a maximum difference of less than $3 \mathrm{MPa}$. Indeed, fictive stress gradient is almost linear below one millimeter and averaging stresses on the layer has very little effect. However, by artificially raising the polishing step, differences between these two incremental methods increase as shown in Fig. 12. Indeed the Savaria-Bridier-Bocher hypothesis of averaging stresses is a better assumption than considering the top value of the polished pocket as the Lambdatech method does. For the discussion on parameter influence that follows, Lambdatech results are not plotted so as to obtain clearer figures.

\section{Discussion on Stress Relaxation Correction Methods}

FEA methods are based on assumptions that have been summed-up [18] some of which have already been discussed by the same authors [20]. Here, the assumption of the independence of the correction regarding the stress profile is tested on numerical methods. The elastic stress relaxation assumption and the influence of the size of the polishing pocket on both the ME analytical methods and the numerical methods are then discussed.

The influence of residual stress fictive profile on numerically corrected results has been studied with modification of the friction coefficient from 0.1 to 0 or to 0.2 . The results are illustrated in Fig. 13. Whereas step-by-step methods are quite robust in relation to changes in fictive profile, Pedersen-Hansson is less stable especially if a change of sign occurs in the profile which is the case with the 0.2 friction coefficient. The proportional correction is then overestimated as the proportion of released fictive stress $\sigma_{p}$ is very large in comparison to the real proportion of stress.

Step-by-step methods attenuate this problem as a change in residual stress sign only affects one correction step and not the entire correction process as it does with the $\mathrm{PH}$ direct proportional correction method. Maximum
Fig. 12 XRD raw and corrected results with a $1 \mathrm{~mm}$ polishing step

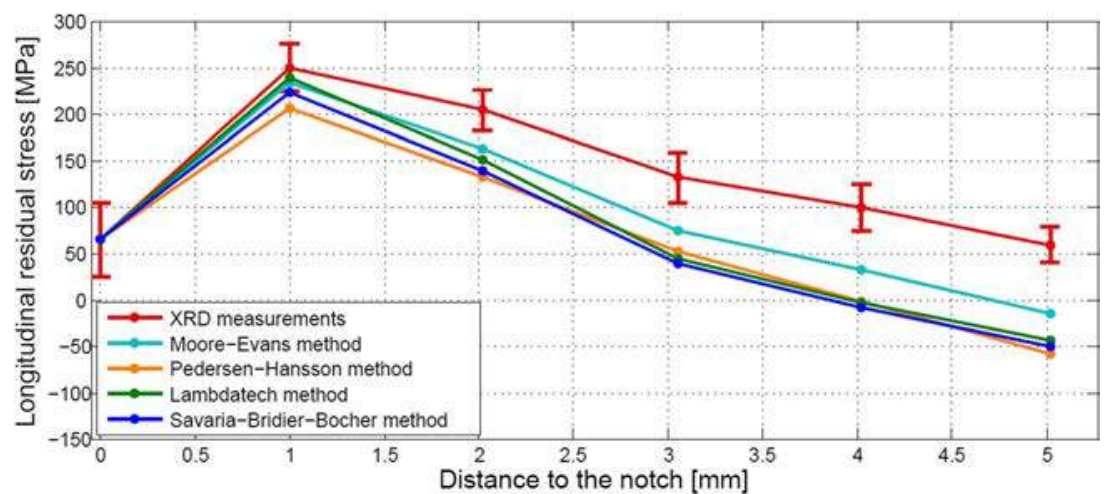


Fig. 13 Influence of fictive stress profile on Pedersen-Hansson method (a) and Savaria-BridierBocher method (b) a)

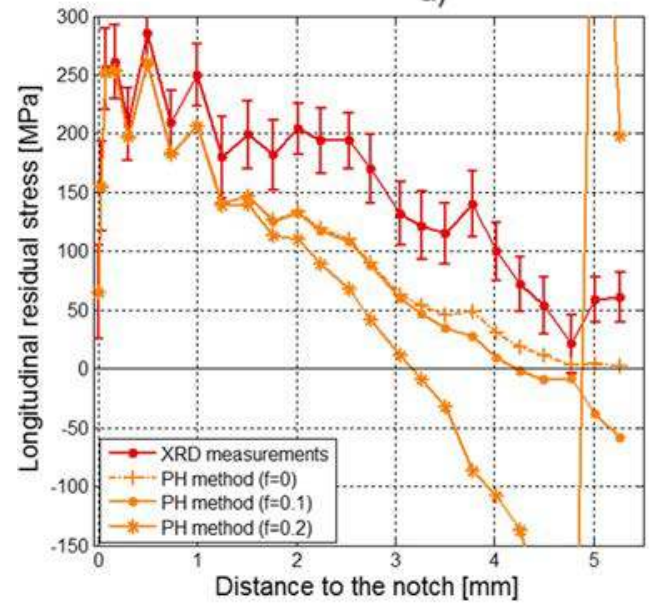

b)

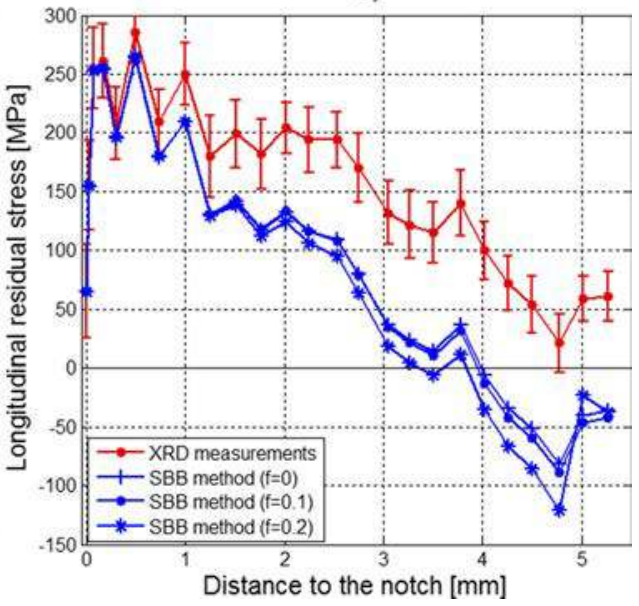

variation of SBB corrected results with the stress profile is $\pm 20 \mathrm{MPa}$ from the results obtained using the reference fictive profile.

Before studying the influence of plasticity, it is important to note that if any numerical correction is applied to ideal measurements, i.e. to the diagonal of the $\frac{\sigma^{f}}{}$ matrix obtained with $f=0.1$, it can be demonstrated from equation (2) to equation (7) that the initial numerical RS profile before polishing will be obtained if the supposed stress evolution of each method is checked. This is also true with ME correction if geometry fits one of the proposed cases.

Thus, to study the influence of the plasticity, a new FEA is computed using the elasto-plastic material behavior during polishing simulation and recording the computed stress on the top of each polished layer. This profile is compared to the "elastic" measurement, i.e. to the diagonal of the $\sigma^{f}$ matrix and results can be seen in Fig. 14. It shows that neglecting plasticity leads to an over-estimation of up to $35 \mathrm{MPa}$ of the evolution of stresses during polishing and that plasticity has no effect after $4 \mathrm{~mm}$.
Numerical correction methods can also be applied to the numerical results obtained using plastic behavior during polishing and compared to the reference solution that is obtained if stress relaxation is elastic, i.e. the numerical predicted RS field. Results are also shown in Fig. 14 and the difference between the corrected stress profiles and the FEA RS field is less than $20 \mathrm{MPa}$. It is interesting to note that $\mathrm{PH}$ exhibits no difference with FEA RS field after $4 \mathrm{~mm}$, i.e. if measured profiles are the same whereas incremental correction methods are influenced by the error made in the first $4 \mathrm{~mm}$ even in the section where plasticity has no effect.

The same procedure is applied with ME methods except that the ME corrected stress profile obtained neglecting plasticity is not the FEA RS profile but differs because the flat plate hypothesis is not verified in this study. The difference between ME correction of the numerical measurements performed with elastic behavior and the ME correction curve of measurements obtained using plastic behavior is up to $30 \mathrm{MPa}$. As the method uses the integral of the stress in the previously removed material, results are also influenced by
Fig. 14 Influence of plasticity on stress correction methods

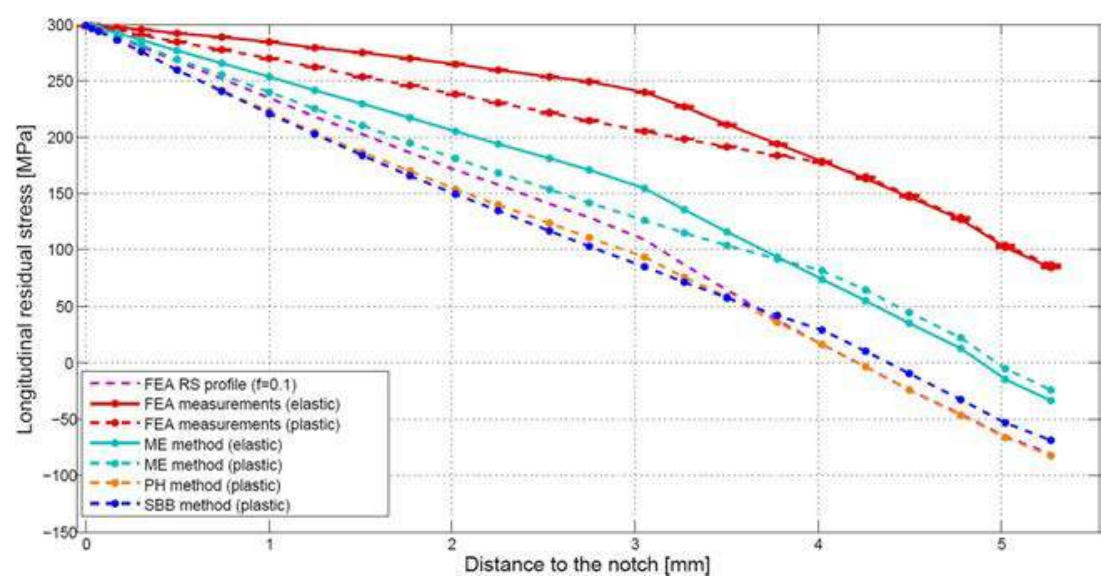


Fig. 15 Influence of the size of the polishing pocket $(\mathrm{PH}$ results are equal to SBB results)

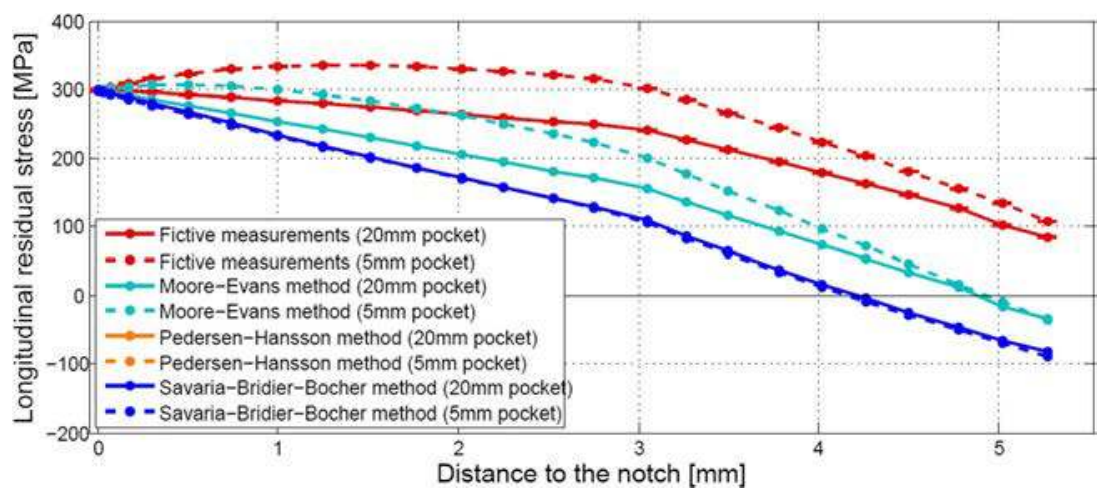

plasticity after $4 \mathrm{~mm}$ as for the Lambdatech or SBB method even though numerical measurements are not.

The previous curves also indicate that the flat plate hypothesis used in that case induces an underestimation of the ME correction of the numerical profiles of up to $60 \mathrm{MPa}$. This value depends on the polishing pocket. A large $20 \mathrm{~mm}$ width polishing pocket in comparison to most industrial applications was used in this work to avoid polishing edge effects such as rounded bottom, due to less salty solution circulation. Stress relaxation edge effects can also occur with local plasticity around pocket corners. Such polishing effects would be difficult to represent numerically.

To study the influence of the size of the polishing pocket, the relaxed stress profile to correct is the numerical stress prediction at the surface of the polishing pocket at each polishing step. Besides the $20 \mathrm{~mm}$ width pocket that has been employed, a second FEA with a $5 \mathrm{~mm}$ width polishing pocket has been simulated.

The two FEA measurement profiles are plotted in Fig. 15 with corrected profiles. It shows that a $5 \mathrm{~mm}$ width polishing pocket leads to less relaxation, and thus higher measured stress.

All numerically corrected results are equals to the FEA RS prediction as these methods take into account the geometry of the polishing pocket and are practiced in the ideal case where the measurements to correct are the diagonal of the $\frac{\sigma^{f}}{\text { matrix. }}$ However, ME corrected result difference with FEA RS prediction is up to $100 \mathrm{MPa}$ with the $5 \mathrm{~mm}$ width pocket whereas it was $60 \mathrm{MPa}$ with the $20 \mathrm{~mm}$ width pocket.

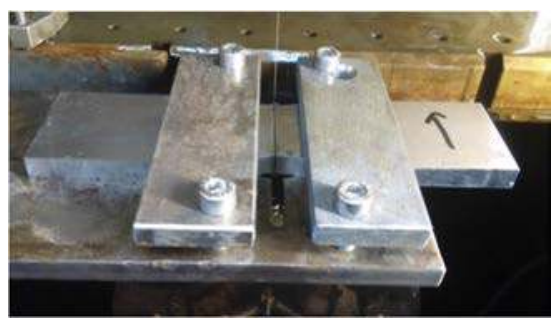

Fig. 16 Part clamping during wire electro-discarding machine cutting
In conclusion, it is more interesting to make a wide polishing when correcting XRD results as it will more easily respect correction method hypothesis in the measurement zone than to make a small polishing to minimize stress relaxation as practiced when no correction is applied.

Therefore, stress correction methods are effective even to several millimeters. The Savaria-Bridier-Bocher is the most robust numerical method available. Method uncertainties due to plasticity or fictive profile influence are estimated to be $\pm 40 \mathrm{MPa}$ in that case and had to be added to initial XRD scattering. If FEA is not available, Moore-Evans analytical method can give satisfying results but special care as to the polishing strategy should be taken to respect these analytical method assumptions.

\section{Contour Method Measurements}

\section{Measurement Conditions}

The contour method [21] is a relaxation-based method that gives a 2D-map of the normal stress component for a cut face. It is thus a destructive method. Besides the complete high resolution cartography of the normal stress, it is a fast and easy-to-access method. The principle of the method is to cut into two parts, to measure the displacement of the two cut faces due to relaxation of stresses and to impose this displacement field in an FEA of one initially undeformed half to obtain

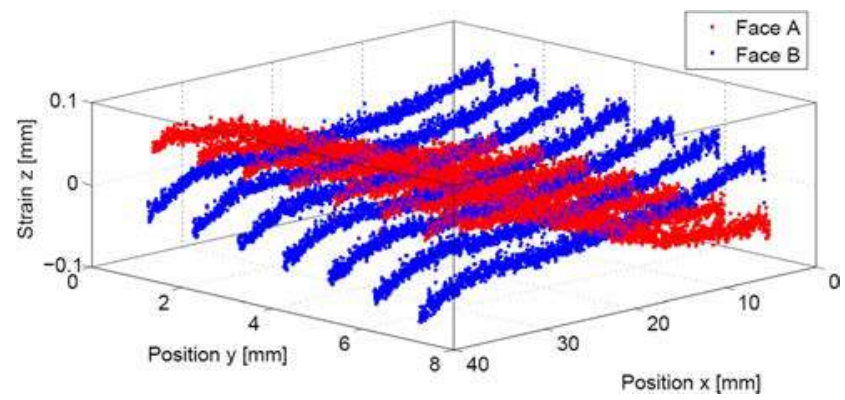

Fig. 17 Raw measured profile of the two cut faces 


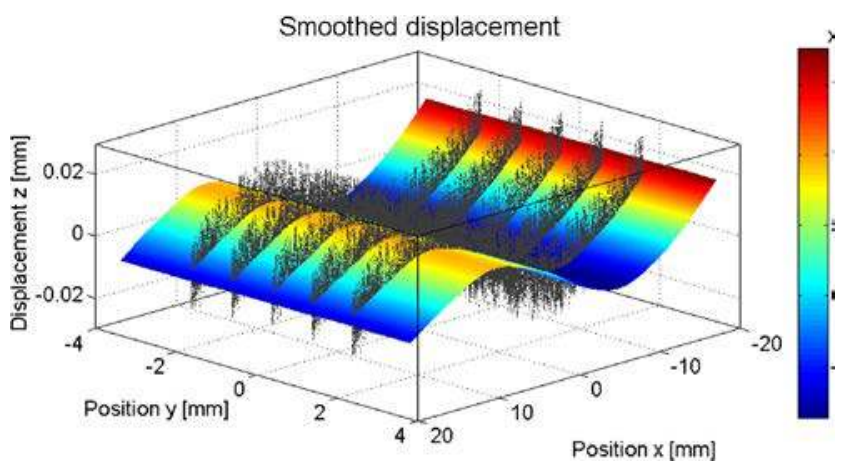

Fig. 18 Smoothed reference displacement field and post- processed data points

the stress field on the whole cut face. In the FEA calculation, macroscopic elastic constants measured during the loadunload test were used $(\mathrm{E}=204,000 \mathrm{MPa}$ and $v=0.3)$.

The part is cut in a symmetry plane using a wire electrodiscarding machine, in order to avoid stress modification due to the cutting process itself and to minimize the cut width. The part is clamped tightly to avoid distortion during cutting [8], as shown in Fig. 16. A $0.25 \mathrm{~mm}$ diameter wire was used at a speed rate of $0.1 \mathrm{~mm} / \mathrm{s}$. The part was cut from one notch to the other through the $38 \mathrm{~mm}$ width. A cut through the $7.2 \mathrm{~mm}$ thickness, from one face to the other would have been more appropriate as the 2D symmetry could have helped with the wire entry/exit issues. A lower cut speed or a thinner wire could have led to less data noise [22] but were not available.

An Altimet ${ }^{\circledR}$ confocal profilometer was used to measure cut face displacement. This fast optical method has an axial accuracy of $0.09 \mu \mathrm{m}$ allowing 25,912 measured points to be obtained. Seven line profiles were made (1 $\mathrm{mm} \mathrm{X}$ resolution) and a $2 \mu \mathrm{m} \mathrm{Z}$ resolution was used. Raw measured profiles of both faces are plotted in Fig. 16 Part clamping during wire electro-discarding machine cutting Fig. 17.

\section{Post-Processing}

Raw data cannot be used directly for several reasons:

- As cut faces are measured separately, they are not in the same coordinate system,

Fig. 19 Cartography of contour results compared to FEA
- Edge effects occur due to cutting and measuring processes,

- Data resolution is not the same as FEA resolution thus interpolation is necessary,

- Data are noisy and using them without smoothing would lead to strong local effects that do not represent the real stress field.

Post-processing is performed as follows. First, outliers are removed based on the distance to the surface. Then, data rotations and translations are applied to help the Iterative Closest Point (ICP) algorithm [23] that is used next to adjust data and FEA mesh in the same coordinate system. FEA mesh spacing was $50 \mu \mathrm{m}$ in $\mathrm{Z}$ direction and $0.9 \mathrm{~mm}$ in $\mathrm{X}$ direction. To avoid measurement edge effects during the post-processing steps of interpolation and smoothing, data and mesh nodes under a distance $d$ from the edge are deleted. The interpolation is performed on the remaining mesh using a closest neighbor algorithm and data are smoothed using cubic splines via Matlab® csaps function [24]. Finally, the smoothed displacement field is extrapolated to the edges using cubic splines.

This post-processing methodology strongly depends on two parameters that have a significant influence on the stress field obtained: $d$, is the distance that includes edge effects and $p$, the cubic spline smoothing parameter of the csaps function that varies from 0 to 1 . A reference post-processing was chosen with parameters $\mathrm{d}=1 \mathrm{~mm}$ and $p=0.005$, which depends on the number of data points (here, 25,912 in total). The displacement field obtained is plotted in Fig. 18 with postprocessed data points.

\section{Results}

Contour results of the reference post-processing allow the observation of the longitudinal stress on the whole cut surface. These results are plotted in Fig. 18 Smoothed reference displacement field and post- processed data points.

Figure 19, and compared to FEA (section "study configuration"). Both results present a good correlation although an important difference exists on the subsurface stress extrema locations. To estimate uncertainties due to the post-
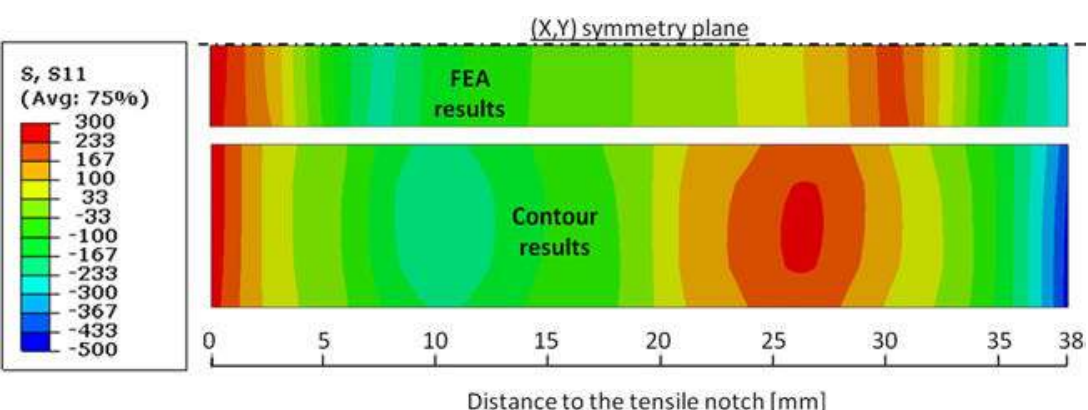
Fig. 20 Contour results compared to XRD, ND and FEA

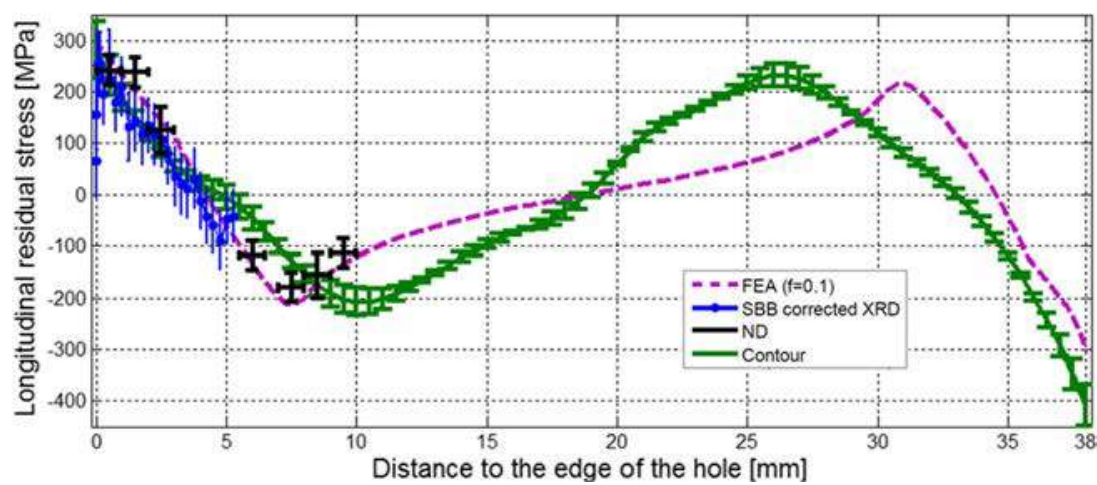

processing method, limit values of the $d$ and $p$ parameters were set as upper and lower reasonable thresholds and nine computations were carried out using the combinations of the 3 values (reference and limits) of the 2 most influential parameters. The nine stress profiles obtained in the middle section of the part were averaged and are presented with the standard deviation in Fig. 20 and compared to XRD, ND and FEA. It is important to note that the averaged stress profile has a maximum deviation of $12 \mathrm{MPa}$ from the chosen post-processed profile $(\mathrm{d}=1, p=0.005)$ which is not plotted for the sake of clarity.

Contour results indicate a surface stress of $300 \mathrm{MPa}$, respectively -460 $\mathrm{MPa}$, at the tensile notch, respectively at the compressive notch. Subsurface extrema of $200 \mathrm{MPa}$ are measured around $10 \mathrm{~mm}$ from the notches whereas ND measurements indicate the same value at $7-8 \mathrm{~mm}$ from the notches. Biggest standard deviation is concentrated on the edges $( \pm 55 \mathrm{MPa})$ where the method is less accurate due to the cutting and measurement edge effects. Otherwise, stress variation with post-processing is less than $\pm 20 \mathrm{MPa}$ at the sub-surface extrema and $\pm 10 \mathrm{MPa}$ on the linear parts.

\section{Conclusion}

The RS of a bent notched specimen were validated through strain measurements, FEA and ND stress measurements. ND performed accurate measurements with a standard deviation of $20 \mathrm{MPa}$ but has an important gauge volume regarding the stress gradient and is not an easy-to-access measurement method.

Two destructive stress measurement techniques that imply material cutting were employed on this bent notched steel specimen to point out the capabilities of each method. XRD has a small gauge volume due to limited X-Ray penetration to the first $10 \mu \mathrm{m}$ requiring polishing to be performed to obtain sub-surface measurements. Due to stress redistribution because of this material removal, XRD measurements needed to be corrected. Four methods were employed and all showed good agreement with ND measurements. The stress difference between the four methods was lower than $30 \mathrm{MPa}$ which is low regarding the fact that stress correction is more than $120 \mathrm{MPa}$ at $5 \mathrm{~mm}$ away from the notch which is the farthest distance reached in this study. Stress correction was greater than measurement scattering after polishing one mm below the surface.

The Moore and Evans method which is the simplest method does not require prior estimation of the residual stress field but uses strong geometrical assumptions unlike numerical stress correction methods. The incremental methods (Lambdatech and Savaria-Bridier-Bocher) give very similar results in the configuration studied and are more robust regarding the supposed stress field than the Pedersen-Hansson method. Uncertainty due to the stress profile is about $20 \mathrm{MPa}$ with incremental methods.

All four methods require an elastic stress relaxation which is not really the case. The ME method is more sensitive to plasticity. The difference between a reference profile and corrected results obtained from ideal FEA measurements taking into account plasticity during stress relaxation can be up to $60 \mathrm{MPa}$ with ME methods. With numerical methods, uncertainty due to plasticity during stress relaxation is lower than $20 \mathrm{MPa}$.

Comparison between analytical and numerical correction methods has highlighted the interest in using wide polishing. Indeed, as correction is used, it is not necessary to minimize stress relaxation. A wide polishing helps to satisfy the correction method hypothesis.

Finally, the contour method was used. This relaxation method is not sensitive to micro-stresses as diffraction methods are and thus offers a complementary measurement. Combining XRD and CM is interesting as they are easy-toaccess, with a low gauge volume adapted to measuring steep gradients and whereas the contour method can measure the complete cartography of a face, it lacks accuracy at the edges, where XRD offers accurate measurements.

$\mathrm{CM}$ results are influenced by post-processing processing procedure and a specific procedure is applied here by averaging the results obtained with influential parameters set to limit values. This procedure allows the uncertainty of the postprocessing procedure to be estimated. Stress variation obtained with this procedure is about $55 \mathrm{MPa}$ at the edges and $20 \mathrm{MPa}$ in the rest of the specimen. 
Acknowledgments The financial support of DGA/MRIS (Mission for Scientific Research and Innovation) is gratefully acknowledged. The authors would also like to thank the Laboratoire Léon Brillouin of the CEA Saclay for the beamtime at line G 5-2.

\section{References}

1. McCLUNG RC (2007) A literature survey on the stability and significance of residual stresses during fatigue. Fatigue Fract Eng Mater Struct 30(3):173-205

2. Withers PJ (2007) Residual stress and its role in failure. Rep Prog Phys 70(12):2211

3. Schajer GS, Ruud CO (2013) Overview of residual stresses and their measurement. Practical residual stress measurement methods, Schajer GS, Ed. John Wiley \& Sons, Ltd 1-27

4. Perenda J, Trajkovski J, Žerovnik A, Prebil I (2015) Residual stresses after deep rolling of a torsion bar made from high strength steel. J Mater Process Technol 218:89-98

5. Epp J, Surm H, Hirsch T, Hoffmann F (2011) Residual stress relaxation during heating of bearing rings produced in two different manufacturing chains. J Mater Process Technol 211(4):637-643

6. Pagliaro P, Prime MB, Robinson JS, Clausen B, Swenson H, Steinzig M, Zuccarello B (2010) Measuring inaccessible residual stresses using multiple methods and superposition. Exp Mech 51(7):1123-1134

7. Toparli MB, Fitzpatrick ME, Gungor S (2015) Determination of multiple near-surface residual stress components in laser peened aluminum alloy via the contour method. Metall Mater Trans A 46(9):4268-4275

8. Xie P, Zhao H, Wu B, Gong S (2015) Evaluation of residual stresses relaxation by post weld heat treatment using contour method and $\mathrm{x}$ ray diffraction method. Exp Mech 55(7):1329-1337

9. Elmesalamy A, Francis JA, Li L (2014) A comparison of residual stresses in multi pass narrow gap laser welds and gas-tungsten arc welds in AISI 316 L stainless steel. Int J Press Vessels Pip 113:49_ 59

10. Scholz J (2002) Experimental analysis and modelling of the mechanical behaviour of an unstable austenitic stainless steel, Paris 6
11. Voce E (1955) A practical strain-hardening function. Metallurgica 51:219-226

12. Armstrong PJ, Frederick CO, Central Electricity Generating Board (1966) A mathematical representation of the multiaxial Bauschinger effect

13. Hauk V (1997) Structural and residual stress analysis by nondestructive methods A2 - s structural and residual stress analysis by nondestructive methods, Amsterdam: Elsevier Science B.V., iii

14. Prévey P (1986) X-ray diffraction residual stress techniques. ASM, 380-392

15. Moore MG, Evans WP (1958) Mathematical correction for stress in removed layers in x-ray diffraction residual stress analysis, SAE International, Warrendale, PA, SAE Technical Paper 580035

16. Pedersen TF, Hansson ILH (1989) Finite element calculations for correction of residual stress profiles of coated and uncoated materials measured by X-ray diffraction. NDT Int 22(6):347-352

17. Lambda Research (1996) Finite element correction for stress relaxation in complex geometries

18. Savaria V, Bridier F, Bocher P (2012) Computational quantification and correction of the errors induced by layer removal for subsurface residual stress measurements. Int J Mech Sci 64(1):184-195

19. Murray CE, Cevdet Noyan I (2013) Applied and residual stress determination using x-ray diffraction. Practical residual stress measurement methods, Schajer GS, Ed. John Wiley \& Sons, Ltd, 139161

20. Savaria V, Monajati H, Bridier F, Bocher P (2015) Measurement and correction of residual stress gradients in aeronautical gears after various induction surface hardening treatments. J Mater Process Technol 220:113-123

21. Prime MB (2000) Cross-sectional mapping of residual stresses by measuring the surface contour after a cut. J Eng Mater Technol 123(2):162-168

22. De Wald AT, Prime MB (2013) The contour method. practical residual stress measurement methods. Wiley, West Sussex, pp 109138

23. Kjer WJHM (2010) Evaluation of surface registration algorithms for PET motion correction

24. MATLAB and Statistics Toolbox Release (2012b) The MathWorks, Inc., Natick, Massachusetts, United States. Csaps function, Cubic smoothing spline 